PROCEEDINGS OF THE

AMERICAN MATHEMATICAL SOCIETY

Volume 135, Number 11, November 2007, Pages 3755-3764

S 0002-9939(07)08953-8

Article electronically published on August 15, 2007

\title{
PLANAR FINITELY SUSLINIAN COMPACTA
}

\author{
ALEXANDER BLOKH, MICHA£ MISIUREWICZ, AND LEX OVERSTEEGEN
}

(Communicated by Alexander N. Dranishnikov)

\begin{abstract}
We show that a planar unshielded compact set $X$ is finitely Suslinian if and only if there exists a closed set $F \subset \mathbb{S}^{1}$ and a lamination $\sim$ of $F$ such that $F / \sim$ is homeomorphic to $X$. If $X$ is a continuum, the analogous statement follows from Carathéodory theory and is widely used in polynomial dynamics.
\end{abstract}

\section{INTRODUCTION}

By a domain we mean a connected open subset of the plane $\mathbb{C}$. A planar compact set $X$ is unshielded if there exists a complementary domain $U$ with $\bar{U} \supset X$ (below we always assume that $U$ contains infinity). Julia sets of polynomials (or of expanding polymodials, see [1, 2]) are unshielded. Below by an unshielded set we always mean a planar unshielded compact set.

We look at unshielded sets "from infinity." That is, let $X$ be a continuum, $U$ be as above, and $\mathbb{D}_{\infty}=\mathbb{C} \backslash \overline{\mathbb{D}}$, where $\mathbb{D}$ is the open unit disk in $\mathbb{C}$. There exists a conformal isomorphism (called a Riemann mapping) $\Psi: \mathbb{D}_{\infty} \rightarrow U$ and, by a result of Carathéodory, $X$ is locally connected if and only if $\Psi$ extends to a continuous function $\bar{\Psi}: \overline{\mathbb{D}_{\infty}} \rightarrow \bar{U}$. To state an important corollary of this result we need the following definition. Consider an equivalence relation $\sim$ on a closed subset $F$ of the unit circle $\mathbb{S}^{1} \subset \mathbb{C}$ with the following properties [6, 9]; cf. [1] (usually $F=\mathbb{S}^{1}$ ):

(L1) $\sim$ is closed: the graph of $\sim$ is a closed set in $F \times F$;

(L2) $\sim$ is unlinked: if $t_{1} \sim t_{2} \in F$ and $t_{3} \sim t_{4} \in F$, but $t_{2} \not t_{3}$, then the straight line segments in $\mathbb{C}$ with endpoints $t_{1}, t_{2}$ and $t_{3}, t_{4}$ are disjoint;

(L3) each equivalence class of $\sim$ is totally disconnected.

Call $\sim$ a lamination of $F$. In the situation above, with an unshielded continuum $X$, set $\psi=\left.\bar{\Psi}\right|_{\mathbb{S}^{1}}$ and define an equivalence $\sim_{\psi}$ on $\mathbb{S}^{1}$ by $x \sim_{\psi} y$ if and only if $\psi(x)=\psi(y)$. Then it is easy to see that the equivalence $\sim_{\psi}$ is a lamination defined on $\mathbb{S}^{1}$ and $X$ is homeomorphic to the quotient space $\mathbb{S}^{1} / \sim_{\psi}$. This yields the following theorem.

Received by the editors January 4, 2006 and, in revised form, September 8, 2006.

2000 Mathematics Subject Classification. Primary 54F15, 54D05, 37F10.

Key words and phrases. Finitely Suslinian, unshielded, locally connected, lamination.

The first author was partially supported by NSF grant DMS 0456748.

The second author was partially supported by NSF grant DMS 0456526 .

The third author was partially supported by by NSF grant DMS 0405774 . 
Theorem 1.1. Let $X$ be an unshielded continuum. If $X$ is locally connected, then there exists a lamination $\sim$ of $\mathbb{S}^{1}$ such that $X$ is homeomorphic to the quotient space $\mathbb{S}^{1} / \sim$.

The converse to this theorem is also true. We will prove it in the next section.

The case when $X$ is locally connected is the best case scenario for our investigation because then the structure of $X$ is closely related to that of $\mathbb{S}^{1}$. Our aim is to obtain a similar result without the assumption of connectivity of $X$. To see what unshielded compacta are similar to unshielded locally connected continua we need the following definition.

Definition 1.2. A compact metric space $X$ is finitely Suslinian (FS) if for each $\varepsilon>0$, any collection of pairwise disjoint continua in $X$ of diameter larger than $\varepsilon$ is finite (we call such compacta FS-sets).

Note that by the definition any FS-set is compact.

The next lemma shows that the notion of an unshielded FS-set is a generalization of the notion of a locally connected unshielded continuum.

Lemma 1.3 ([3, Lemma 2.7]). An unshielded continuum $Y$ is locally connected if and only if it is FS.

Now we are ready to state our main result, namely Theorem 1.4 which is analogous to Theorem 1.1 and its converse; its main applications are in the field of complex dynamics and will be discussed in forthcoming papers.

Theorem 1.4. Let $X$ be an unshielded compact set. Then $X$ is FS if and only if there exists a closed set $F \subset \mathbb{S}^{1}$ and a lamination $\sim$ of $F$ such that $X$ is homeomorphic to the quotient space $F / \sim$.

The authors are indebted to the referee for a careful reading of the original manuscript which resulted in several improvements.

\section{REDUCTION}

In this section we reduce Theorem 1.4 to the following theorem, which is the main technical result of the paper.

Theorem 2.1. Any unshielded FS-set is contained in an unshielded FS-continuum.

To prove one implication in Theorem 1.4, we have to show that if $X$ is an unshielded FS-set, then there exist a closed set $F \subset \mathbb{S}^{1}$ and a lamination $\sim$ of $F$ such that $X$ is homeomorphic to $F / \sim$. If Theorem 2.1 holds, then there exists an unshielded FS-continuum $Y \supset X$. Then by Lemma 1.3 and Theorem 1.1 there exists a lamination $\sim$ of $\mathbb{S}^{1}$ with $Y=\mathbb{S}^{1} / \sim$. Let $p: \mathbb{S}^{1} \rightarrow \mathbb{S}^{1} / \sim$ be the corresponding quotient map, and $F=p^{-1}(X)$. Then $F$ is closed and $F / \sim$ is homeomorphic to $X$. This proves the implication, provided that Theorem 2.1 holds.

In order to prove the other implication in Theorem 1.4, assume that for a set $X$ there is a closed set $F \subset \mathbb{S}^{1}$ and a lamination $\sim$ on $F$ such that $X$ is homeomorphic to $F / \sim$. We want to prove that then $X$ is FS. Let us start with a simple lemma that follows immediately from the fact that continuous maps of metric compact spaces are uniformly continuous.

Lemma 2.2. Any set homeomorphic to an FS-set is itself an FS-set. 
Now we prove a theorem that is essentially the converse to Theorem 1.1 .

Theorem 2.3. Let $\sim$ be a lamination of $\mathbb{S}^{1}$. Then any set $X \subset \mathbb{C}$ homeomorphic to the quotient space $\mathbb{S}^{1} / \sim$ is locally connected and FS.

Proof. Let $L$ be the union of $\sim$-hulls, i.e., the convex hulls of $\sim$-classes in $\mathbb{D}$. Define an extension $\simeq$ of $\sim$ onto $\mathbb{C}$ as follows [6]: a $\simeq$-class is a $\sim$-hull or a point of $\mathbb{C} \backslash L$. The quotient space $K=\overline{\mathbb{D}} / \simeq$ is embedded in the quotient space $\mathbb{C} / \simeq$. Denote the interior of $K$ by $Q$. Let $p: \mathbb{C} \rightarrow \mathbb{C} / \simeq$ be the quotient map. Then $\left.p\right|_{\mathbb{C} \backslash \mathbb{D}}: \mathbb{C} \backslash \overline{\mathbb{D}} \rightarrow(\mathbb{C} / \simeq) \backslash K$ and $\left.p\right|_{\mathbb{D} \backslash L}: \mathbb{D} \backslash L \rightarrow Q$ are homeomorphisms. The set $Z=p\left(\mathbb{S}^{1}\right)=p(L)$ is the boundary of $K$ in $\mathbb{C} / \simeq$; clearly, $Z$ is homeomorphic to $\mathbb{S}^{1} / \sim$. Observe that $K$ and $Z$ are compact, connected and locally connected because $p: \overline{\mathbb{D}} \rightarrow K$ is continuous. Note also that $p^{-1}(z)$ is a nonseparating plane continuum for each $z$. By a theorem of Moore [10, $\mathbb{C} / \simeq$ is homeomorphic to the plane. By construction the set $Z$ is unshielded in the plane $\mathbb{C} / \simeq$, and the fact that $Z$ is FS follows from Lemma 1.3. Since $X$ is homeomorphic to $Z$ and by Lemma 2.2, $X$ is locally connected and FS.

Now we can prove the second implication in Theorem 1.4. Assume that $X$ is an unshielded compact set and there exists a closed set $F \subset \mathbb{S}^{1}$ and a lamination $\sim$ of $F$ such that $X$ is homeomorphic to the quotient space $F / \sim$. If $F=\mathbb{S}^{1}$, then by Theorem 2.3. $X$ is FS. Assume that $F \neq \mathbb{S}^{1}$. Extend the equivalence $\sim$ onto the entire unit circle $\mathbb{S}^{1}$ by declaring that a point in $\mathbb{S}^{1} \backslash F$ is equivalent only to itself. This creates a lamination $\sim$ on $\mathbb{S}^{1}$. By Theorem 2.3 the continuum $Y=\mathbb{S}^{1} / \sim$ is FS. Obviously, the quotient space $F / \sim$ is homeomorphic to a compact subset $R$ of $Y$. Since $X$ is homeomorphic to $F / \sim$, it is also homeomorphic to $R$. A compact subset of an FS-set is clearly an FS-set, so $R$ is an FS-set, and thus, by Lemma 2.2. $X$ is an FS-set itself. This proves the implication and completes the reduction of Theorem 1.4 to Theorem 2.1

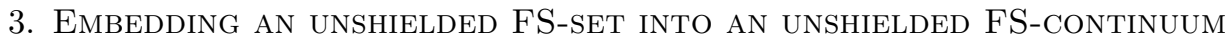

In this section we prove Theorem 2.1, using an inductive construction.

Given a compact planar set $A$ and $\varepsilon>0$ let $B(A, \varepsilon)$ be the union of all open $\varepsilon$ balls centered at points of $A$. Open sets $U$ and $V$ are called strongly disjoint if their closures are disjoint. Given a set $X$ we denote its boundary by $\partial X=\bar{X} \backslash \operatorname{int}(X)$. By a Jordan disk we mean the bounded component of $\mathbb{C} \backslash P$, where $P \subset \mathbb{C}$ is a Jordan curve. Given a compactum $Y \subset \mathbb{C}$, the unbounded component $U$ of $\mathbb{C} \backslash Y$ is well defined. Call the set $T(Y)=\mathbb{C} \backslash U$ the topological hull of $Y$. To get the topological hull of a compactum one adds to this compactum all bounded components of its complement ("fill-in"). We have $\operatorname{diam}(Y)=\operatorname{diam}(T(Y))$ and the set $T(Y)$ is also compact. If a compactum $X$ is unshielded, then we can say more: $T(A) \cap T(B)=\emptyset$ for any two distinct components $A$ and $B$ of $X$, and $X$ is the boundary of $T(X)$. Finally, if $W$ is open, $K \subset W$ is a continuum and for some $\varepsilon>0$ and for each $x \in W \backslash T(K)$ there exists an $\operatorname{arc} A_{x} \subset W$ joining $x$ to $K$ with $\operatorname{diam}\left(A_{x}\right)<\varepsilon$, then $W$ is called an $\varepsilon$-arc-neighborhood of $K$.

Lemma 3.1. Let $X \subset \mathbb{C}$ be an unshielded FS-set and let $\eta>0$. Then for every component $K$ of $X$ there exists a Jordan disk $U$ such that $K \subset T(K) \subset U \subset$ $B(T(K), \eta)$ and $\partial U$ is disjoint from $X$. 
Proof. Set $X^{\prime}=T(X)$. Fix a component $K$ of $X$. By [5], there exists a sequence $\varepsilon_{n} \searrow 0$ such that for each $n$, each component of the boundary of $B\left(X^{\prime}, \varepsilon_{n}\right)$ is a singleton or an arc or a Jordan curve. One of those closed curves has to be the boundary of a Jordan disk $U_{n}$ containing $K$.

Set $K^{\prime}=\bigcap_{n} \overline{U_{n}}$. Clearly, $T(K) \subset K^{\prime}$. To prove the reverse inclusion, observe that the boundaries of $B\left(X^{\prime}, \varepsilon_{n}\right)$ and $B\left(X^{\prime}, \varepsilon_{n+1}\right)$ are disjoint, so $U_{n+1} \subset U_{n}$. Therefore $K^{\prime}$ is a continuum as an intersection of the descending sequence of continua. Suppose that there is a point $x \in K^{\prime} \backslash X^{\prime}$. Then it is contained in the unbounded component of $\mathbb{C} \backslash X$, so it can be connected with $\infty$ by an arc $A$ disjoint from $X^{\prime}$. The distance between $A$ and $X^{\prime}$ is positive, so if $n$ is sufficiently large, then $A$ is disjoint from $\partial U_{n}$. Thus, $x \notin \overline{U_{n}}$, a contradiction. This proves that $K^{\prime} \subset X^{\prime}$. Thus, $K^{\prime}$ is a connected subset of $X^{\prime}$ containing $T(K)$. Since $T(K)$ is a connected component of $X^{\prime}$, we get $K^{\prime}=T(K)$. Therefore, if $n$ is sufficiently large, then $K \subset U_{n} \subset B(T(K), \eta)$.

By the definition of $\partial U_{n}$, it is disjoint from $X^{\prime}$. This completes the proof.

Lemma 3.2. Let $X \subset \mathbb{C}$ be an unshielded FS-set, $Y$ a closed subset of $X$ such that every component of $X$ intersecting $Y$ is contained in $Y$, and let $\varepsilon \geq \eta>0$. Assume that every component of $Y$ has diameter smaller than $\varepsilon$. Then there exist strongly disjoint Jordan disks $U_{1}, \ldots, U_{n}$ such that $Y \subset \bigcup_{i=1}^{n} U_{i} \subset B(T(Y), \eta)$ and for $i=1, \ldots, n$ the diameter of $U_{i}$ is less than $3 \varepsilon$, the boundary of $U_{i}$ is disjoint from $X$ and $U_{i} \cap Y \neq \emptyset$.

Proof. By Lemma 3.1 for every component $K$ of $Y$ there exists a Jordan disk $U$ such that $K \subset U \subset B(T(K), \eta)$ and $\partial U$ is disjoint from $X$. This gives us an open cover of $Y$, so we can choose from it a finite subcover $V_{1}, \ldots, V_{k}$. We can modify each $V_{i}$ by moving slightly its boundary in $\mathbb{C} \backslash T(X)$, so that the resulting disks $V_{i}^{\prime}$ still satisfy $K_{i} \subset V_{i}^{\prime} \subset B\left(T\left(K_{i}\right), \eta\right)$ for the appropriate components $K_{i}$ of $Y$, and so that $\partial V_{i}^{\prime}$ is disjoint from $X$ and $\partial V_{i}^{\prime} \cap \partial V_{j}^{\prime}$ is finite if $i \neq j$. Let $U_{i}^{\prime}, i=1, \ldots, n$, be the bounded components of $\mathbb{C} \backslash \bigcup_{j=1}^{k} \partial V_{j}^{\prime}$ whose intersection with $Y$ is nonempty. Then all $U_{i}^{\prime}$ are Jordan disks. Their boundaries are contained in $\bigcup_{j=1}^{k} \partial V_{j}^{\prime}$, so they are disjoint from $X$. Each $U_{i}^{\prime}$ is contained in some $V_{j}^{\prime} \subset B\left(T\left(K_{i}\right), \eta\right)$, so $U_{i}^{\prime} \subset B(T(Y), \eta)$ and $\operatorname{diam}\left(U_{i}^{\prime}\right)<\varepsilon+2 \eta \leq 3 \varepsilon$. Now we can replace each $U_{i}^{\prime}$ by a slightly smaller Jordan disk $U_{i}$ such that $\overline{U_{i}} \subset U_{i}^{\prime}, U_{i} \cap X=U_{i}^{\prime} \cap X$ and $\partial U_{i} \cap X=\emptyset$. Then the disks $U_{1}, \ldots, U_{n}$ satisfy the assertions of the lemma.

Now we can prove a stronger version of Lemma 3.1

Lemma 3.3. Let $X \subset \mathbb{C}$ be an unshielded FS-set and let $\varepsilon>0$. Then for every component $K$ of $X$ there exists a Jordan disk $U$ such that $U$ is an $\varepsilon$-arc-neighborhood of $T(K)$ and $\partial U$ is disjoint from $X$.

Proof. By Lemma 1.3 each component $K$ is locally connected, so the Riemann mapping $\Psi: \mathbb{D}_{\infty} \rightarrow \mathbb{C} \backslash T(K)$ extends to a continuous map $\bar{\Psi}: \overline{\mathbb{D}_{\infty}} \rightarrow \overline{\mathbb{C} \backslash T(K)}$. Restricted to $D_{R} \cap \mathbb{D}_{\infty}$, where $D_{R}$ is the disk of radius $R>1$ centered at the origin, this map is uniformly continuous. Therefore for a given $\delta>0$ there exists $\xi>0$ such that the diameter of the ray piece $\left\{\bar{\Psi}(z): z=t z_{0} \in \mathbb{C}, 1 \leq t \leq 1+\xi\right\}$ is smaller than $\delta$ for any $z_{0}$ of modulus 1 . Then the set $V_{\delta}=T(K) \cup \Psi(\{z \in \mathbb{C}: 1<|z|<1+\xi\})$ is a Jordan disk with the boundary $\Psi(\{z \in \mathbb{C}:|z|=1+\xi\})$ and is a $\delta$-arc-neighborhood of $T(K)$. 
Since $X$ is an FS-set, we may choose $\delta<\varepsilon / 7$ so small that every component of $X$ that meets $\partial V_{\delta}$ has diameter less than $\varepsilon / 7$. Denote the union of all components of $X$ that meets $\partial V_{\delta}$ by $X_{K}$. Then $X_{K}$ is a closed subset of $X$ and each component of $X_{K}$ has diameter less that $\varepsilon / 7$. Let $\eta>0$ be smaller than the distance between $X_{K}$ and $K$. By Lemma 3.2, there exist strongly disjoint Jordan disks $U_{1}, \ldots, U_{n}$ such that $X_{K} \subset \bigcup_{i=1}^{n} U_{i} \subset B\left(T\left(X_{K}\right), \eta\right)$ and for $i=1, \ldots, n$ the diameter of $U_{i}$ is less than $3 \varepsilon / 7$ and the boundary of $U_{i}$ is disjoint from $X$. For each $i$, since $U_{i} \subset B\left(T\left(X_{K}\right), \eta\right)$, we have $\overline{U_{i}} \cap K=\emptyset$. Let $U$ be the component of $\mathbb{C} \backslash\left(\bigcup_{i=1}^{n} \partial U_{i} \cup \partial V_{\delta}\right)$ that contains $K$. By Theorem 4, p. 512 of [8], $U$ is a Jordan disk. We claim that $U$ is the required $\varepsilon$-arc-neighborhood of $T(K)$. To see this choose $x \in U \backslash T(K)$. Since $x \in V_{\delta}$, there exists an arc $A \subset V_{\delta}$ of diameter less than $\varepsilon / 7$ joining $x$ to $K$. If $A$ meets a $\overline{U_{i}}$, we can modify it by using a piece of $\partial U_{i}$. Using the triangle inequality it is easy to see that doing this for all $\overline{U_{i}}$ that $A$ meets results in a new arc $A^{\prime}$ of diameter less than $\varepsilon / 7+6 \varepsilon / 7=\varepsilon$ as required. By the construction, $\partial U$ is disjoint from $X$.

Lemma 3.4. Let $X$ be an unshielded FS-set contained in a Jordan disk $U$ and let $K_{0}, \ldots, K_{m}$ be some components of $X$. Then for each $\varepsilon>0$ there exists a finite cover $\left\{U_{0}, \ldots, U_{n}\right\}(n \geq m)$ of $X$ by strongly disjoint Jordan disks and components $K_{m+1}, \ldots, K_{n}$ of $X$ such that for each $i \in\{0, \ldots, n\}, K_{i} \subset U_{i} \subset \overline{U_{i}} \subset U$ and $U_{i}$ is an $\varepsilon$-arc-neighborhood of $T\left(K_{i}\right)$. Moreover, for each component $K$ of $X$ such that $K \notin\left\{K_{0}, \ldots, K_{n}\right\}, \operatorname{diam}(K)<\varepsilon / 2$.

Proof. Let $X, U$ and $K_{0}, \ldots, K_{m}$ be as specified above. Fix $\varepsilon>0$. Since $X$ is $\mathrm{FS}$, there exist $k \geq m$ and components $K_{m+1}, \ldots, K_{k}$ such that $\operatorname{diam}(K)<\varepsilon / 3$ for any component $K$ of $X$ which is not one of $K_{0}, \ldots, K_{k}$. By Lemma 3.3 there exist strongly disjoint Jordan disks $U_{0}, \ldots, U_{k}$ such that $U_{i}$ is an $\varepsilon$-arc-neighborhood of $T\left(K_{i}\right), \overline{U_{i}} \subset U$ and $\partial U_{i}$ is disjoint from $X$ for $i=0, \ldots, k$.

Set $Y=X \backslash \bigcup_{i=0}^{k} U_{i}$. Then $Y$ is a closed subset of $X$ and its distance from $\left(X \cap \bigcup_{i=0}^{k} U_{i}\right) \cup(\mathbb{C} \backslash U)$ is positive. Therefore by Lemma 3.2 there exist Jordan disks $U_{k+1}, \ldots, U_{n}$ of diameter less than $\varepsilon$ such that $Y \subset \bigcup_{i=k+1}^{n} U_{i}, \overline{U_{i}} \subset U$, $\partial U_{i}$ is disjoint from $X$ and $U_{i} \cap Y \neq \emptyset$ for $i=k+1, \ldots, n$ and all Jordan disks $U_{0}, \ldots, U_{k}, U_{k+1}, \ldots, U_{n}$ are strongly disjoint. To complete the proof, we choose components $K_{i}$ of $X, i=k+1, \ldots, n$, with $K_{i} \subset U_{i}$.

Given a Jordan disk $U$ and two points $a, b \in U$ there exists a unique $\operatorname{arc} S(a, b)$ of shortest arc length joining $a, b$ in $\bar{U}[4$. We may, and from now on do, assume that all Jordan disks have a piecewise linear boundary. Under this assumption there exists a unique shortest arc $S(a, b) \subset \bar{U}$ between any two points $a, b \in \bar{U}$ and the length $|S(a, b)|$ of $S(a, b)$ is finite. We call $S(a, b)$ the $s$-arc (between $a$ and $b$ in $U$ ). By [4] the family of s-arcs is continuous in the following sense: if $a_{i} \rightarrow a, b_{i} \rightarrow b$ and all these points belong to $U$, then $S\left(a_{i}, b_{i}\right)$ converge to $S(a, b)$ in the Hausdorff topology and $\left|S\left(a_{i}, b_{i}\right)\right| \rightarrow|S(a, b)|$. Hence, given two disjoint compact subsets $A, B$ of $U$ there is an arc of shortest length between a point of $A$ and a point of $B$ : just take a sequence of pairs of points $a_{i}, b_{i}$ such that $\left|S\left(a_{i}, b_{i}\right)\right|$ converges to the infimum of the lengths over all arcs connecting a point of $A$ with a point of $B$, and use the continuity. Thus we can talk of s-arcs between compacta; in particular, given a point $x \in U$ and a Jordan disk $V \subset \bar{V} \subset U$ we can find an arc (contained in $\bar{U}$ ) of shortest length from $x$ to $\bar{V}$.

Similarly, one can consider arcs of smallest diameter between two points in $U$. Given two points $a, b \in U$, call an arc with endpoints $a$ and $b$ a $d$-arc (between $a$ and 
$b$ in $U)$ if its diameter is the smallest possible among all arcs connecting $a$ and $b$ in $\bar{U}$. By [7], if $a, b \in X \subset \bar{U}$ and $X$ is a continuum, then $\operatorname{diam}(X) \geq \operatorname{diam}(S(a, b))$. Hence the s-arc between $a$ and $b$ is a d-arc between $a$ and $b$. The case of arcs between sets is analogous to the above. Given two compacta $A, B \subset U$ call an arc with the endpoints in $A, B$ a $d$-arc (between $A$ and $B$ ) if it has the smallest diameter among all such arcs. To show that d-arcs between $A$ and $B$ exist, choose sequences $a_{i} \in A, b_{i} \in B$ so that $a_{i} \rightarrow a \in A, b_{i} \rightarrow b \in B$ and $\operatorname{diam}\left(S\left(a_{i}, b_{i}\right)\right)$ converges to the infimum of the diameters of arcs connecting points of $A$ and $B$. Then by [4] the arcs $S\left(a_{i}, b_{i}\right)$ converge to the arc $S(a, b)$ in the Hausdorff topology, and it is easy to see that $\operatorname{diam}\left(S\left(a_{i}, b_{i}\right)\right) \rightarrow \operatorname{diam}(S(a, b))$. Thus, $S(a, b)$ is a d-arc between $A$ and $B$. An upper bound on the diameter of an s-arc between a point in $U$ and a Jordan disk in $U$ is established below.

Lemma 3.5. Let $U$ be a Jordan disk, $V \subset \bar{V} \subset U$ be a Jordan disk, $x \in U$ be a point, $\delta$ be the diameter of a d-arc between $x$ and $\bar{V}$, and let $S(x, y)$ be an s-arc between $x$ and $\bar{V}$. Then $\operatorname{diam}(S(x, y)) \leq 9 \delta$.

Proof. Choose a d-arc $S(x, z)$ between $x$ and $\bar{V}$ of diameter $\delta$, such that $S(x, z) \cap$ $\bar{V}=\{z\}$. We may assume that $x \notin \bar{V}$ and $z \neq y$. Observe that since $S(x, y)$ is an s-arc between $x$ and $\bar{V}$, we have $S(x, y) \cap \bar{V}=\{y\}$. Fix $\varepsilon>\delta$; we will show that $\operatorname{diam}(S(x, y))<9 \varepsilon$. Draw the circle $P$ centered at $x$ of radius $\varepsilon$. If $S(x, y) \subset T(P)$, then $\operatorname{diam}(S(x, y)) \leq 2 \varepsilon<9 \varepsilon$ as desired. Thus we may assume that $S(x, y)$ is not a subset of $T(P)$.

By the uniqueness of shortest arcs, there is a point $q$ such that $S(x, y) \cap S(x, z)=$ $S(x, q)$. Moreover, $\overline{S(x, y) \backslash S(x, q)}=S(q, y)$ and $\overline{S(x, z) \backslash S(x, q)}=S(q, z)$. Since $S(x, z) \subset T(P)$, we have $q \in T(P)$. Choose an arc $R \subset \partial V$ connecting $y$ and $z$ and consider the Jordan curve $A=S(q, y) \cup R \cup S(q, z)$.

Clearly, $A \subset \bar{U}$ and $\operatorname{int}(T(A)) \subset U$ is a Jordan disk. Let $W$ be the component of $\operatorname{int}(T(P)) \cap \operatorname{int}(T(A))$ containing $q$ (and hence $S(q, z)$ ) in its boundary (by Theorem 4, p. 512 of $[\underline{8}$, all components of $\operatorname{int}(T(P)) \cap \operatorname{int}(T(A))$ are Jordan disks). Note that $\partial W \subset \bar{A} \subset \bar{U}$. Let $B \subset \partial W \backslash\{z\}$ be the minimal subarc of $\partial W$ that connects $q$ to $\partial V$. Then $B \subset S(q, y) \cup P$. Since $S(q, y)$ is an s-arc between $q$ and $V$, then $|B| \geq|S(q, y)|$. Hence the parts of $S(q, y)$ that are not in $B$ have total length at most $2 \pi \varepsilon$. In particular, all parts of $S(x, y)$ outside $T(P)$ have total length at most $2 \pi \varepsilon$. Therefore by the triangle inequality $\operatorname{diam}(S(x, y)) \leq 2 \varepsilon+2 \pi \varepsilon<9 \varepsilon$. Since $\varepsilon$ can be chosen arbitrarily close to $\delta$, we get $\operatorname{diam}(S(x, y)) \leq 9 \delta$.

We use Lemma 3.5 in the proof of the next lemma.

Lemma 3.6. Let $U$ be a Jordan disk containing the closures of strongly disjoint Jordan disks $V_{0}, V_{1}, \ldots, V_{i}$ and let $V_{i+1}, \ldots, V_{n}$ be points on $\partial U$. Choose $\varepsilon>0$ so that $U$ is an $\varepsilon$-arc-neighborhood of $\overline{V_{0}}$ and $\operatorname{diam}\left(V_{k}\right)<\varepsilon, k=1, \ldots, n$. Then there exist disjoint trees $T_{1}, \ldots, T_{m}, m<\infty$, such that $\operatorname{diam}\left(T_{j}\right)<20 \varepsilon$, exactly one point of each $T_{j}$ is in $\overline{V_{0}}$, and $\left(\bigcup_{j=1}^{m} T_{j}\right) \cap \overline{V_{i}}$ is a singleton for each $i=1, \ldots, n$.

Proof. By the remarks preceding Lemma 3.5, there is an s-arc $A_{i}^{\prime}=S\left(a_{i}, b_{i}\right)$ between $\overline{V_{i}}$ and $\overline{V_{0}}\left(a_{i} \in \overline{V_{i}}, b_{i} \in \overline{V_{0}}\right)$. If $x \in A_{i}^{\prime} \cap A_{j}^{\prime}$, then $S\left(x, b_{i}\right)$ is the arc in $A_{i}^{\prime}$ from $x$ to $b_{i}, S\left(x, b_{j}\right)$ is the arc of $A_{j}^{\prime}$ from $x$ to $b_{j}$, and these arcs have the same length. Hence the arc, say, $S\left(a_{i}, x\right) \cup S\left(x, b_{j}\right)$ is still an s-arc between $a_{i}$ and $\overline{V_{0}}$ (the original s-arc is $A_{i}^{\prime}$ ). Endow $A_{i}^{\prime}$ with the order $a_{i}<b_{i}$ and use interval notation for subarcs of $A_{i}^{\prime}$. Inductively define s-arcs $A_{i}, 1 \leq i \leq n$, between $a_{i}$ and 
$\overline{V_{0}}$ so that each $G_{k}=\bigcup_{j=1}^{k} A_{j}, 1 \leq k \leq n$, is the union of disjoint trees, each of which intersects $\overline{V_{0}}$ at one point. We can do this as follows. First we set $A_{1}=A_{1}^{\prime}$. Suppose $A_{1}, \ldots, A_{k}$ have been defined; choose the smallest point $x_{k+1}$ on $A_{k+1}^{\prime}$ which belongs to $\overline{V_{0}} \cup \bigcup_{j=1}^{k} A_{j}$, denote by $B$ the unique arc in $G_{k}$ joining $x_{k+1}$ to $\overline{V_{0}}$ (such an arc is unique by induction), and set $A_{k+1}=\left[a_{k+1}, x_{k+1}\right] \cup B$. It is now easy to verify the inductive assumptions, which completes the construction.

Let $C$ be a component of $G_{n}, C \cap \overline{V_{0}}=\{z\}$. By the construction, $C$ is the union of s-arcs between a point in $U$ and $\overline{V_{0}}$. By Lemma 3.5 each such arc has diameter at most $9 \varepsilon$, so $\operatorname{diam}(C) \leq 18 \varepsilon$. Since some $\operatorname{arcs} A_{i}$ and $\overline{V_{j}}, j \neq i$, may intersect, to complete the proof we modify $G_{n}$. Choose strongly disjoint Jordan disks $V_{i}^{\prime} \supset \overline{V_{i}}$ of diameters less than $\varepsilon$. For each $i$ choose a Jordan disk $D_{i} \subset V_{i}$ such that $\partial D_{i} \cap \partial V_{i}=\overline{D_{i}} \cap G_{n}=\left\{a_{i}\right\}$ and also a Jordan disk $D_{i}^{\prime} \subset \overline{D_{i}^{\prime}} \subset V_{i}^{\prime}$ containing $V_{i}$ and such that $\partial D_{i}^{\prime} \cap \partial V_{i}=\left\{a_{i}\right\}$. Then we can use an isotopy in $\overline{V_{i}^{\prime}}$ which expands $D_{i}$ onto $D_{i}^{\prime}$ and is the identity on $\partial V_{i}^{\prime} \cup\left\{a_{i}\right\}$. Repeating this for all $i$, we construct the desired union of trees. Since each change we made is confined to a set of diameter less than $\varepsilon$, the diameter of each tree does not change by more than $2 \varepsilon$.

To prove Theorem 2.1 we apply Lemma 3.6, the inductive construction is close to 2. For brevity and to follow the terminology of [2, below we call Jordan disks bubbles. Choose a bubble $U_{0} \supset X, R>\operatorname{diam}\left(U_{0}\right)$, and a component $K_{0}$ of $X$. Set $\delta_{n}=4^{-n}$. By Lemma 3.4 we can find a finite cover $\left\{U_{0}^{1}, \ldots, U_{m(1)}^{1}\right\}$ of $X$ by strongly disjoint bubbles and components $K_{i}^{1} \subset U_{i}^{1}$ of $X$ satisfying the conditions of Lemma 3.4 with $\varepsilon=\delta_{1}$ and $K_{0}=K_{0}^{1}$. Thus, after the first step we have $m(1)+1$ components $K_{i}^{1}, 0 \leq i \leq m(1)$ of $X$ with strongly disjoint $\delta_{1}$-arc-neighborhoods of their topological hulls: the bubbles $U_{i}^{1}, 0 \leq i \leq m(1)$. By Lemma 3.6 the sets $\overline{U_{i}^{1}}, 1 \leq i \leq m(1)$ can be connected to $\overline{U_{0}^{1}}$ by means of pairwise disjoint trees of diameters less than $R$. On sets $\partial U_{i}^{1}, 0 \leq i \leq m(1)$ we mark finitely many points at which those trees intersect them (there will be at most $m(1)$ marked points on $\partial U_{0}^{1}$ and one marked point on each $\left.\partial U_{i}^{1}, 1 \leq i \leq m(1)\right)$. Finally, by Lemma 3.4 all other components of $X$ (not belonging to $\left\{K_{0}^{1}, \ldots, K_{m(1)}^{1}\right\}$ ) are of diameter less than $\delta_{1} / 2$. Let us denote the union of all connecting trees and all sets $U_{i}^{1}, 0 \leq i \leq m(1)$ by $T_{1}$. The set $T_{1}$ can be thought of as a tree in which some points are replaced by bubbles. Observe that by Lemma 3.6 the connecting trees do not penetrate the bubbles.

Apply the same construction to the pairs $\left(U_{i}^{1}, K_{i}^{1}\right), 0 \leq i \leq m(1)\left(K_{i}^{1}\right.$ and $U_{i}^{1}$ replace $K_{0}=K_{0}^{1}$ and $U_{0}$, and the constant is $\delta_{2}$, not $\delta_{1}$ ). The difference is that now we have bubbles and finitely many points on the boundary of each $U_{i}^{1}$. Still, Lemma 3.6 is applicable. Thus, inside each bubble of the first generation we construct bubbles of the the second generation each of which is a $\delta_{2}$-arc-neighborhood of the appropriate component of $X$. Moreover, components $K_{0}^{1}, \ldots, K_{m(1)}^{1}$ chosen on the previous step remain on the list of chosen components of the second generation, and some bubbles of the second generation are $\delta_{2}$-arc-neighborhoods of the topological hulls of those components. The trees with bubbles, constructed inside the bubbles of the first generation, replace the bubbles of the first generation and are then added to already existing trees. To sum it all up, once the construction is applied inside each $U_{i}^{1}$, we complete the second step which gives rise to the set 
$T_{2} \subset T_{1}$ which is also a tree with bubbles. Now apply the same arguments inside each bubble of the second generation with the new constant $\delta_{3}$, etc. In the end we get the infinite intersection $Z=\bigcap T_{n}$. Then $Z$ is a nonseparating continuum. Put $Y=\partial Z$. Then $y$ is an unshielded continuum. Let us now establish some properties of the construction.

Property A. We have $X \subset Z$.

Proof. It follows from the fact that $X \subset T_{n}$ for any $n$.

If $K \subset X$ is a component of $X$, denote by $U_{K}^{n}$ the bubble of the $n$-th generation containing $K$.

Property B. If $K$ is a component of $X$, then $\bigcap_{n} \overline{U_{K}^{n}}=\bigcap_{n} U_{K}^{n}=T(K)$.

Proof. By the construction, $\bigcap_{n} \overline{U_{K}^{n}}=\bigcap_{n} U_{K}^{n}$. Set $B=\bigcap_{n} \overline{U_{K}^{n}}$. Then by the construction $T(K) \subset B$. On the other hand, no point outside $T(K)$ belongs to $B$ because for any $\varepsilon>0$ from some time on the sets $U_{K}^{n}$ are contained in $\varepsilon$-balls around $K$. Hence $B=T(K)$.

Property C. We have $X \subset Y$.

Proof. Let $K$ be any component of $X$ and let $U_{K}^{n}$ be the bubble of the $n$-th generation containing $K$. By Property $\mathrm{B}, \bigcap_{n} \overline{U_{K}^{n}}=\bigcap_{n} U_{K}^{n}=T(K)$. Since $X$ is unshielded, $K=\lim \partial U_{K}^{n}$. It follows from the construction that $\partial U_{K}^{n} \subset \overline{\mathbb{C} \backslash Z}$ for every $n$. Hence $K \subset \partial Z=Y$.

Call a pair $(U, K)$ which appears in the construction (on the step $n$ ) a building pair (of generation $n$ ). Recall that $T(X)$ denotes the union of the topological hulls of all components of $X$ and set $A=Y \backslash T(X)$. Represent $A$ as a countable union of open (homeomorphic to $(0,1))$ arcs as follows. Let $(U, K)$ be a building pair of generation $n,(V, K)$ and $(W, L)$ be building pairs of generation $n+1, W \subset U$. Then there is a unique arc $I \subset T_{n+1}$ connecting $W$ and $V$. Outside $W$ this arc is extended on each step so that its endpoint approaches $K$. Finitely many points on $\partial U$ (coming from the previous step) are connected to $V$ similarly. These sets, connecting to $K$ either bubbles of the next generation (contained in $U$ ), or the finitely many points chosen on $\partial U$, are called partial connectors (of generation $n$ ). On the other hand, a similar process takes place inside $W$ where the partial connector towards $L$ is being constructed. The entire inductively constructed set connecting $L$ and $K$ is said to be a connector. Clearly, there are countably many connectors, and $A$ is the union of all of them. By the construction, a connector is the union of two partial connectors (above, one partial connector extends towards $K$, and the other one towards $L$ ). Hence the claim that all connectors are arcs follows from Property D below.

Property D. Let $(U, K)$ be a building pair of generation $n,(W, L)$ be a building pair of generation $n+1, W \subset U$ and $K \neq L$. Then the following estimates hold.

(1) $\operatorname{diam}(W) \leq 4^{-n}$.

(2) Let $I$ be a partial connector between $W$ and $K$, or between one of the finitely many points chosen on $\partial U$, and $K$. Then $\bar{I}$ is a closed arc, $\operatorname{diam}(I)<\frac{80}{3} 4^{-n}$, and if $I$ is a partial connector between $W$ and $K$, then $\operatorname{diam}(I \cup W)<\frac{83}{3} 4^{-n}$. 
Proof. (1) By the construction $\operatorname{diam}(L)<\delta_{n} / 2$. By Lemma 3.4 and the construction, for each point $x \in W$ there is a point $y \in L$ such that $|x-y| \leq \delta_{n+1}$. Hence $\operatorname{diam}(W) \leq \delta_{n} / 2+2 \delta_{n+1}=4^{-n}$.

(2) By the construction and Lemma 3.6 the partial connector $I$ between $W$ and $K$ (or between an appropriate point on $\partial U$ and $K$ ) is the concatenation of disjoint (except endpoints where two consecutive arcs meet) arcs of diameters at most $20 \delta_{n}, 20 \delta_{n+1}, \ldots$ Hence $\operatorname{diam}(I) \leq 20\left(\delta_{n}+\delta_{n+1}+\ldots\right)=\frac{80}{3} 4^{-n}$. Since the endpoint of $I$ belongs to $K$, it does not belong to any of the arcs that we concatenate. Therefore $\bar{I}$ is a closed arc. Together with (1) this implies that $\operatorname{diam}(I \cup W)<\frac{83}{3} 4^{-n}$ and completes the proof.

Property E. If $C \subset Y$ is a continuum, then its intersection with a component $K$ of $X$ is a continuum too.

Proof. Let $C_{n}$ be the intersection of $C$ with the bubble of $n$-th generation containing $K$. Then, by the definition of $T_{n}$, the set $C_{n}$ is connected. The sequence of continua $\left(C_{n}\right)_{n=1}^{\infty}$ is descending, so its intersection $C \cap T(K)$ is a continuum. Since $C \subset Y$ and $Y \cap T(K)=K$, we have $C \cap T(K)=C \cap K$.

To prove Theorem 2.1 we need to show that $Y$ is FS. Suppose that for some $\varepsilon>0$ there are infinitely many disjoint continua $C_{n} \subset Y$ with diameter greater than $\varepsilon$. Choose $m>1$ so that $\delta_{m}=4^{-m}<\frac{3 \varepsilon}{332}$. Consider $T_{m}$. As we know $T_{m}$ is a tree with finitely many bubbles; hence only finitely many $C_{n}$ 's are not contained in one of those bubbles. Thus we may assume that for a building pair $(U, K)$ of generation $m$ all $C_{n}$ 's are contained in $U$.

Let us estimate the diameters of the $C_{n}$ 's. Since the $C_{n}$ 's are disjoint, their intersections with $K$ are disjoint too. By Property E each intersection $K \cap C_{n}$ is a continuum, and since $K$ is FS we see that $\operatorname{diam}\left(C_{n} \cap K\right) \rightarrow 0$. Hence we may assume that no $C_{n}$ is contained in $K$ and that $\operatorname{diam}\left(C_{n} \cap K\right)<\varepsilon / 2$. Now, it follows from the construction that the set $(Y \cap U) \backslash K$ is the union of sets, each of which is contained in a set of the form listed in Property D (2). That is, it is either contained in the union of a bubble $V \subset U$ of generation greater than $m$ and a partial connector between $K$ and $V$, or it is contained in a partial connector between one of the finitely many chosen points on $\partial U$ and $K$. By Property D every set in the list is of diameter at most $\frac{83}{3} 4^{-m}$. By the triangle inequality, this implies that $\operatorname{diam}\left(C_{n}\right) \leq \frac{166}{3} 4^{-m}+\varepsilon / 2<\varepsilon$ (the last inequality follows from the fact that $\left.4^{-m}<\frac{3 \varepsilon}{332}\right)$, a contradiction. Hence, $Y$ is finitely Suslinian.

\section{REFERENCES}

1. A. Blokh, C. Cleveland, and M. Misiurewicz, Expanding polymodials, in: Modern Dynamical Systems and Applications, (M. Brin, B. Haselblatt, and Y. Pesin, eds.), Cambridge University Press, Cambridge (2004), pp. 253-270. MR2090774 (2006d:37076)

2. A. Blokh, C. Cleveland, and M. Misiurewicz, Julia sets of expanding polymodials, Ergodic Theory and Dynam. Syst. 25 (2005), 1691-1718. MR2183289 (2006h:37071)

3. A. Blokh and L. Oversteegen, Backward stability for polynomial maps, Trans. Amer. Math. Soc. 356 (2004), 119-133. MR2020026 (2005c:37081)

4. R. D. Bourgin and P. L. Renz, Shortest paths in simply connected regions in $\mathbb{R}^{2}$, Adv. Math. 76 (1989), 260-295. MR1013673 (90k:52021)

5. M. Brown, Sets of constant distance from a planar set, Michigan Math. J. 19 (1972), 321-323. MR 0315714 (47:4263)

6. A. Douady, Descriptions of compact sets in $\mathbb{C}$, in: Topological methods in modern mathematics, Publish or Perish, (1993), pp. 429-465. MR1215973 (94g:58185) 
7. P. Fabel, "Shortest" arcs in closed planar disks vary continuously with the boundary, Top. Appl. 95 (1999), 75-83. MR.1691933 (2000d:58016)

8. K. Kuratowski, Topology II, Academic Press, New York, 1968.

9. C. T. McMullen, Complex dynamics and renormalization, Annals of Mathematical Studies 135, Princeton University Press, Princeton, NJ (1994). MR.1312365 (96b:58097)

10. R. L. Moore, Concerning upper semicontinuous collections of compacta, Trans. Amer. Math. Soc. 27 (1925), 416-428. MR.1501320

11. W. Thurston, The combinatorics of iterated rational maps, Preprint (1985).

Department of Mathematics, University of Alabama in Birmingham, University Station, Birmingham, Alabama 35294-2060

E-mail address: ablokh@math.uab.edu

Department of Mathematical Sciences, iUpui, 402 N. Blackford Street, IndianapoLIS, INDIANA 46202-3216

E-mail address: mmisiure@math.iupui.edu

Department of Mathematics, University of Alabama in Birmingham, University Station, Birmingham, Alabama 35294-2060

E-mail address: overstee@math.uab.edu 